\title{
ON THE RADICAL OF A RING WITH MINIMUM CONDITION
}

\author{
D. W. BARNES \\ (received 18 September 1964)
}

The purpose of this note is to establish the following characterisation of the radical:

THEOREM. Let $R$ be a ring with the minimum condition for left ideals. Then the radical of $R$ is the intersection of the maximal nilpotent subrings of $R$.

We prove first the following lemmas, assuming throughout that $R$ is a ring with minimum condition:

Lemma 1. Suppose $R$ is the direct sum $R_{1} \oplus \cdots \oplus R_{k}$ of the ideals $R_{i}, i=1,2, \cdots, k$. Let $N_{i}$ be a maximal nilpotent subring of $R_{i}(i=1$, $2, \cdots, k)$, and let $N=N_{1} \oplus \cdots \oplus N_{k}$. Then $N$ is a maximal nilpotent subring of $R$. Conversely, if $N$ is a maximal nilpotent subring of $R$, then the $R_{i}$-component

$$
N_{i}=\left\{x \mid x=n-y \in R_{i} \text { for some } n \in N, y \in \sum_{j \neq i} R_{j}\right\}
$$

of $N$ is a maximal nilpotent subring of $R_{i}$, and

$$
N=N_{1} \oplus \cdots \oplus N_{k} .
$$

Proof. Consider the product $a_{1} a_{2} \cdots a_{t}$ of elements $a_{i} \in R$. Each $a_{i}$ is uniquely expressible in the form

Then

$$
a_{i}=b_{i 1}+b_{i 2}+\cdots+b_{i k}, \quad b_{i j} \in R_{j} .
$$

$$
a_{1} a_{2} \cdots a_{t}=b_{11} b_{21} \cdots b_{t 1}+b_{12} b_{22} \cdots b_{t 2}+\cdots+b_{1 k} b_{2 k} \cdots b_{t k}
$$

since $R$ is the direct sum of the ideals $R_{i}, i=1,2, \cdots, k$. Thus $a_{1} a_{2} \cdots a_{t}=0$ if and only if $b_{1 i} b_{2 i} \cdots b_{t i}=0$ for all $i=1,2, \cdots, k$. Thus a subring $S$ of $R$ is nilpotent if and only if for all $i$, the $R_{i}$-component

of $S$ is nilpotent.

$$
S_{i}=\left\{x \mid x=s-y \in R_{i} \text { for some } s \in S, y \in \sum_{j \neq i} R_{j}\right\}
$$

(i) Let $N_{i}$ be a maximal nilpotent subring of $R_{i}(i=1,2, \cdots, k)$, and let $N=N_{1} \oplus \cdots \oplus N_{k}$. Then $N$ is a nilpotent subring of $R$. Suppose $S$ 
is a nilpotent subring of $R$ and $S \geqq N$. Then the component $S_{i} \geqq N_{i}$ and is nilpotent. Since $N_{i}$ is maximal nilpotent in $R_{i}$, we must have $S_{i}=N_{i}$. But

$$
S \leqq \sum_{i=1}^{k} S_{i}=\sum_{i=1}^{k} N_{i}=N
$$

Therefore $S=N$ and $N$ is maximal nilpotent in $R$.

(ii) Let $N$ be a maximal nilpotent subring of $R$. Then the components $N_{i}$ of $N$ are nilpotent. Suppose $R_{i} \geqq S_{i} \geqq N_{i}$ and $S_{i}$ is nilpotent, $i=1,2, \cdots, k$. Then $S=S_{1} \oplus \cdots \oplus S_{k}$ is a nilpotent subring of $R$ and $S \geqq N$. Therefore $S=N$ which implies $S_{i}=N_{i}$. Thus $N_{i}$ is a maximal nilpotent subring of $R_{i}$ and $N=N_{1} \oplus \cdots \oplus N_{k}$.

Lemma 2. Suppose $R$ is simple, non-null. Then there exist maximal nilpotent subrings $U, L$ of $R$ such that $U \cap L=0$.

Proof. $R$ is isomorphic to the ring of endomorphisms of some finitedimensional left vector space over some division ring $D$. From any basis of $V$, we obtain a faithful representation of $R$ by matrices $\left(d_{i j}\right)$ with elements $d_{i j}$ in $D$. If $N$ is any nilpotent subring of $R$, we can choose the basis of $V$ such that every element of $N$ is represented by an upper triangular matrix $\left(d_{i j}\right), d_{i j}=0$ for $i \geqq j$. Clearly the subring $U$ of all elements of $R$ which are represented (for some given basis of $V$ ) by upper triangular matrices is a maximal nilpotent subring of $R$. The subring $L$ of elements represented by lower triangular matrices $\left(d_{i j}\right), d_{i j}=0$ for $i \leqq j$, is also a maximal nilpotent subring of $R$ and $U \cap L=0$.

Lemma 3. Suppose $R$ is semi-simple. Then the intersection of the maximal nilpotent subrings of $R$ is 0 .

Proof. $R$ is the direct sum $S_{1} \oplus \cdots \oplus S_{k}$ of simple non-null ideals $S_{i}$. For each $i$, there exist maximal nilpotent subrings $U_{i}, L_{i}$ of $S_{i}$ such that $U_{i} \cap L_{i}=0$. Put $U=U_{1} \oplus \cdots \oplus U_{k}$ and $L=L_{1} \oplus \cdots \oplus L_{k}$. Then $U, L$ are maximal nilpotent subrings of $R$ and $U \cap L=0$.

LeMma 4. Let $N$ be the radical of $R$ and let $K$ be a subring of $R$. Then $K$ is a maximal nilpotent subring of $R$ if and only if $K \geqq N$ and $K / N$ is a maximal nilpotent subring of $R / N$.

Proor. If $K$ is nilpotent, then so is $(K+N) / N$. But $(K+N) / N$ and $N$ both nilpotent implies that $K+N$ is nilpotent. Thus if $K$ is maximal nilpotent, then $K=K+N$ and therefore $K \geqq N$. Suppose $K \geqq N$. Then $K$ is nilpotent if and only if $K / N$ is nilpotent. Thus $K(\geqq N)$ is maximal nilpotent in $R$ if and only if $K / N$ is maximal nilpotent in $R / N$.

Proof of Theorem. Let $N$ be the radical of $R$, and let $M_{\alpha}$ be the maximal nilpotent subrings of $R$. Then $M_{\alpha} \geqq N$ for all $\alpha$, and 


$$
\left(\bigcap_{\alpha} M_{\alpha}\right) / N=\bigcap_{\alpha}\left(M_{\alpha} / N\right)
$$

But the $M_{\alpha} / N$ are all the maximal nilpotent subrings of the semi-simple ring $R / N$. Therefore

$$
\bigcap_{\alpha}\left(M_{\alpha} / N\right)=0
$$

and therefore

$$
\bigcap_{\alpha} M_{\alpha}=N
$$

\section{Reference}

[1] Artin, E., Nesbitt, C. J. and Thrall, R. M., Rings with minimum condition (University of Michigan Press, Ann Arbor, 1944).

The University of Sydney 